\title{
Multi-k magnetic phases and topological charges in the candidate Weyl semimetal CeAIGe
}

\author{
Jonathan White \\ Paul Scherrer Institute, Villigen, Switzerland; \\ jonathan.white@psi.ch
}

In topological materials science, the aim is to find pronounced phenomena rooted in the concepts of topology in new materials, and harness them for novel and robust functions. Promising materials classes include magnetic materials hosting nanoscale magnetic skyrmions, or Dirac and Weyl semimetals, which are hallmarked by topological invariants in real- or reciprocal spaces, respectively. With recent attention focused on magnetic topological materials, here we consider the question if novel functionalities may be found in systems with electronic and magnetic structures that are both topologically nontrivial, and where they coexist and may be coupled.

In this context, I will present our recent experimental work on the polar tetragonal magnet CeAlGe [1]. This system was predicted recently to be an easy-plane ferromagnetic type-II Weyl semimetal, with the magnetic and electrical properties little-explored. We combine magnetometry, neutron scattering and electrical transport measurements to reveal CeAlGe as a host of incommensuratelymodulated multi- $\boldsymbol{k}$ magnetic phases with a nanometric length-scale. Application of modern magnetic symmetry analysis methods for refining neutron diffraction data reveals the ground state magnetic structure contains topological merons and antimerons, which can be thought of as 'half-skyrmions' carrying half-integer topological charge. While the ground state carries no topological Hall effect, the effect emerges for a phase induced by an intermediate field along the polar c-axis, which may be generated by a magnetic structure containing anti-meron pairs. We discuss the implication for the existence of such magnetic phases in Weyl semimetals and the possibilities for new functionalities.

[1]. P. Puphal et al. and J.S. White, Phys. Rev. Lett. 124, 017202 (2020)

Keywords: magnetism, incommensurate, symmetry, multi-k, meron 